\title{
A FIXED-POINT THEOREM FOR CENTRAL ELEMENTS IN QUASISIMPLE GROUPS
}

\author{
HARVEY I. BLAU
}

(Communicated by Ronald Solomon)

\begin{abstract}
It is shown that with a few exceptions (which are listed), any central element in a quasisimple finite group fixes some conjugacy class of the group under multiplication.
\end{abstract}

Feit and Seitz proved that an outer automorphism of a finite simple group must fuse some distinct conjugacy classes [FS, Theorem C]. Suppose that $G=$ $S\langle\alpha\rangle$, where $S$ is a finite simple group, $\alpha$ is an outer automorphism of $S$ $(S \leq G \leq \operatorname{Aut}(S)$, where $S$ is identified with $\operatorname{Inn}(S))$, and $|G: S|$ is a prime power. Then Feit and Seitz's "non-fixed-point" result, along with Brauer's permutation lemma [I, (6.3.2)], implies that $S$ must be the inertial subgroup in $G$ of some irreducible character of $S$, and hence yields the following "fixed-point theorem":

If $G=S\langle\alpha\rangle$, where $S$ is a finite simple group and $\alpha$ an outer automorphism of $S,|G: S|$ is a prime power, and $\lambda$ is a linear character of $G$, then $\chi \lambda=\chi$ for some $\chi \in \operatorname{Irr}(G)$.

It seems natural to pose a kind of dual to Feit and Seitz's theorem:

If $G$ is a finite quasisimple group (i.e., $G$ is perfect and $G / Z(G)$ is simple), and if $z \in Z(G)$, is there a conjugacy class $C$ of $G$ with $C z=C$ ?

Any central element of any group $G$ permutes the conjugacy classes of $G$ by multiplication; the question merely asks if fixed-points must occur under this action when $G$ is quasisimple. We show in this paper that the answer is almost always yes, and we delineate the cases where the answer is no. Specifically, we prove the following (the notation for simple groups is essentially as in [G] or [CCNPW], and all groups considered here are finite):

Theorem 1. Assume that $G$ is a quasisimple group and let $z \in Z(G)$. Then one of the following holds:

(i) $\operatorname{order}(z)=6$ and $G / Z(G) \cong A_{6}, A_{7}, F i_{22}, \operatorname{PSU}\left(6,2^{2}\right)$, or ${ }^{2} E_{6}\left(2^{2}\right)$;

(ii) $\operatorname{order}(z)=6$ or 12 and $G / Z(G) \cong \operatorname{PSL}(3,4), \operatorname{PSU}\left(4,3^{2}\right)$ or $M_{22}$;

(iii) $\operatorname{order}(z)=2$ or $4, G / Z(G) \cong \operatorname{PSL}(3,4)$, and $Z(G)$ is noncyclic;

(iv) there exists a conjugacy class $C$ of $G$ such that $C z=C$.

Received by the editors December 21, 1992.

1991 Mathematics Subject Classification. Primary 20D06, 20D08; Secondary 20D05, $20 \mathrm{C} 15$.

Key words and phrases. Quasisimple group, simple group, Schur multiplier, conjugacy class. 
Remarks. (1) The classification of the finite simple groups is essential for the proof of the theorem, as it is for the proof of [FS, Theorem C]. Our proof is given in a sequence of lemmas below. The key point occurs early in the proof of Lemma 4, and exploits the following result from the theory of algebraic groups: the number of semisimple conjugacy classes of the universal Chevalley (or twisted Steinberg) cover of a finite simple group of Lie type is a power of the underlying prime characteristic. This allows the application of an easy counting argument to the action of central elements of (different) prime power order on these classes.

(2) Each case of (i)-(iii) arises in a way such that (iv) fails (except that the author does not know whether (iv) fails when $\operatorname{order}(z)=6$ in a six-fold or 12fold cover of $\left.{ }^{2} E_{6}\left(2^{2}\right)\right)$. Every other such configuration where $Z(G)$ is cyclic may be checked in the Atlas [CCNPW], by inspecting the "lifting order rows". When $G$ is the universal cover of $\operatorname{PSL}(3,4)$, an argument similar to that of Lemma 6 below (which uses Lemma 1) shows that exactly six of the twelve elements of order 4 in $Z(G)$ fix a conjugacy class of $G$. If $a$ is one of the other six elements of order 4 in $Z(G)$, then $a\left\langle a^{2}\right\rangle$, of order 2 in $Z\left(G /\left\langle a^{2}\right\rangle\right)$, fixes no conjugacy class of $G /\left\langle a^{2}\right\rangle$.

(3) R. C. Thompson [T] answered the general question of which elements are commutators in the family of quasisimple groups $\operatorname{SL}(n, K), K$ any field; so for those groups the conclusion of Theorem 1 was known.

(4) The theorem is applied, along with [FS, Theorem C], in work of Arad, Erez, Fisman, and the author which examines hypotheses on conjugacy classes or characters of a group which force the group to have a Frobenius-like structure [ABEF].

Throughout the paper, the statement that a central element $z$ "fixes a conjugacy class" simply means that under the group multiplication, $C z=C$ for some conjugacy class $C$. The Schur multiplier of a group $S$ is denoted by $M(S)$. The Schur multipliers of the finite simple groups are all known, and may be found in [G, p. 302] or [CCNPW].

Lemma 1. Let $G$ be a finite group and $z \in Z(G)$. Then $C z=C$ for some conjugacy class $C$ of $G$ if and only if $\sum_{\chi \in \operatorname{Irr}(G)} \chi(z) / \chi(1) \neq 0$.

Proof. By [I, p. 45], $z$ is a commutator $x^{-1} y^{-1} x y$ for some $x, y \in G$ if and only if $\sum_{\chi \in \operatorname{Irr}(G)} \chi(z) / \chi(1) \neq 0$. But $z=x^{-1} y^{-1} x y \Leftrightarrow x z=y^{-1} x y \Leftrightarrow C z=$ $C$, where $C$ is the conjugacy class of $x$.

Remark. The criterion [I, p. 45] may be very useful in determining which elements of groups are commutators. In this paper, however, the special case of Lemma 1 is applied only to covers of PSU $\left(4,3^{2}\right)$ in Lemma 6, and of $\operatorname{PSL}(3,4)$ in the remark above.

Lemma 2. Suppose that $G$ is a group with $Z(G) \cong L \times K$, where $(|L|,|K|)=1$. Assume that $z \in L$ and that $z K$ fixes a conjugacy class of $G / K$. Then $z$ fixes a conjugacy class of $G$.

Proof. The hypotheses imply that there is a conjugacy class $C$ of $G$ such that $C z K=C K$. Hence, $C z k=C$ for some $k \in K$. Since $(|L|,|K|)=1$, there exists an integer $n>0$ with $n \equiv 1(\bmod |L|)$ and $n \equiv 0(\bmod |K|)$. Therefore, $C=C(z k)^{n}=C z$. 
Lemma 3. If $G$ is quasisimple, $G / Z(G) \cong A_{n}$ for some $n>7$ and $z \in Z(G)$, then there exists a conjugacy class $C$ of $G$ with $C z=C$.

Proof. It suffices to assume that $G$ is the universal covering group of $A_{n}$ [G, $4.227,4.228]$. One of the representation groups for $S_{n}$, which we denote as $\widetilde{S}_{n}$, is given by generators $-1, \sigma_{1}, \sigma_{2}, \ldots, \sigma_{n-1}$ and relations

$$
\begin{gathered}
(-1)^{2}=1 ; \quad(-1) \sigma_{j}=\sigma_{j}(-1) ; \quad \sigma_{j}^{2}=-1 ; \\
\left(\sigma_{j} \sigma_{k}\right)^{2}=-1 \quad \text { if }|j-k| \geq 2 ; \quad\left(\sigma_{j} \sigma_{j+1}\right)^{3}=-1 ;
\end{gathered}
$$

where in the natural projection of $\widetilde{S}_{n}$ onto $S_{n},-1 \mapsto 1$ and $\sigma_{j} \mapsto(j j+1)$, the adjacent transposition. Now $G$ is the preimage of $A_{n}$ in $\widetilde{S}_{n}$ ([S1] or [S2]). Thus $z:=-1$ is the only nontrivial element of $Z(G)$. One easily checks that

$$
\sigma_{1} \sigma_{3}(-1)=\sigma_{3} \sigma_{1}=\left(\sigma_{1} \sigma_{3}\right)^{\sigma_{1} \sigma_{5}}
$$

Hence, $C z=C$ where $C$ is the class of $\sigma_{1} \sigma_{3}$ in $G$.

Lemma 4. Let $S$ be a simple group of Lie type (nontwisted or twisted), defined over a field of characteristic $p$, such that $p \nmid|M(S)|$. If $G$ is quasisimple, $G / Z(G) \cong S$ and $z \in Z(G)$, then there is a conjugacy class $C$ of $G$ with $C z=C$.

Proof. The hypothesis implies that the universal covering group $U$ of $S$ is precisely the universal Chevalley or twisted Steinberg cover of $S[G, 4.228$, 4.234]. It suffices to assume $G=U$. Now $G$ is the set of fixed points; under a suitable Frobenius endomorphism, of a simply connected algebraic group [SS, $\S 2]$. Hence, the cardinality of $\mathbf{S}:=$ the set of semisimple $\left(p^{\prime}-\right)$ conjugacy classes of $G$ is $p^{a}$ for some $a>0$, by Corollary 3.11 of Springer and Steinberg [SS].

Suppose that $\operatorname{order}(z)$ is a positive power of some prime $s$ (so $s \neq p$ ). Now $\langle z\rangle$ acts on $\mathbf{S}$ by multiplication. It follows that if $C z \neq C$ for all $C \in \mathbf{S}$ then $s|| \mathbf{S} \mid$, a contradiction. Thus, $C z=C$ for some $C \in \mathbf{S}$. Now $M(S)$ is either a 2-group or a 3-group unless $S \cong \operatorname{PSL}\left(n, p^{m}\right)$ or $\operatorname{PSU}\left(n, p^{2 m}\right)$ for some $n, m>0$ ([G, p. 302] or [CCNPW, p. xvi]), so the result is proved except in those cases.

Let $(S, \varepsilon)$ denote either $\left(\operatorname{PSL}\left(n, p^{m}\right), 1\right)$ or $\left(\operatorname{PSU}\left(n, p^{2 m}\right),-1\right)$. Then $G \cong \mathrm{SL}\left(n, p^{m}\right)$ or $\mathrm{SU}\left(n, p^{2 m}\right)$ and $Z(G) \cong Z_{d}$, cyclic of order $d=$ $\left(n, p^{m}-\varepsilon\right)$. Now $z \in Z(G)$ implies that $z=\lambda I_{n}$, where $\lambda \in G F\left(p^{m}\right)$ or $G F\left(p^{2 m}\right)$ and $\lambda^{d}=1$. So $\operatorname{order}(\lambda)=r$, where $r \mid d$.

Suppose first that either $r$ is odd or $n / r$ is even. Let $g$ be the diagonal matrix which consists of $n / r$ diagonal blocks of the form $\operatorname{diag}\left(\lambda, \lambda^{2}, \ldots, \lambda^{r-1}, \lambda^{r}\right)$. The parity assumptions imply that $\operatorname{det} g=1$, and hence $g \in G$. Let $h$ be the $n \times n$ block diagonal matrix where each block is an $r \times r$ permutation matrix which is a cycle of length $r$. Then $\operatorname{det} h=1, h \in G$, and $g z=g^{h}$. Hence, $C z=C$, where $C$ is the $G$-conjugacy class of $g$.

So we may assume that $r$ is even and $n / r$ is odd. Let $\gamma$ be a square root of $\lambda$ in a suitable extension field of $G F\left(p^{m}\right)$ or $G F\left(p^{2 m}\right)$. Let $M$ be the $r \times r$ companion matrix of the polynomial $X^{r}+1=\prod_{i=0}^{r-1}\left(X-\gamma \lambda^{i}\right)$. So $\operatorname{det} M=1$ and the set of eigenvalues of $M$ is $\left\{\gamma \lambda^{i} \mid 0 \leq i \leq r-1\right\}$. Thus $\left(\lambda I_{r}\right) \cdot M$ and $M$ have the same set of eigenvalues, and hence the same characteristic polynomial $X^{r}+1$. So the semisimple elements $M$ and $\left(\lambda I_{r}\right) \cdot M$ are conjugate in $\operatorname{SL}\left(r, p^{m}\right)$ 
(if $\varepsilon=1$ ) or $\mathrm{SU}\left(r, p^{2 m}\right.$ ) (if $\varepsilon=-1$ ) [SS, (3.15)]. Now if $g$ is the $n \times n$ block diagonal matrix where each block is $M$, then $g$ and $z g$ are conjugate in $G$. Hence in this case as well, $C z=C$ where $C$ is the $G$-conjugacy class of $g$. The lemma is proved.

Lemma 5. Suppose that $G$ is quasisimple and $Z(G) \cong L \times Z_{2} \times Z_{2}$, where $L \cong 1$ or $Z_{3}$. Assume also that there is an automorphism of $G$ which transitively permutes the three involutions in $Z(G)$. Let $z \in Z(G)$ with $\operatorname{order}(z)=2$ or 3 , and suppose that if $\bar{G}$ is any quasisimple homomorphic image of $G$ such that $|Z(\bar{G})|=2$ or 3 , then $\bar{z}$ (the image of $z$ in $\bar{G}$ ) fixes a conjugacy class of $\bar{G}$. Then $z$ fuxes a conjugacy class of $G$.

Proof. If $\operatorname{order}(z)=3$, then the result follows from the hypothesis and Lemma 2 , with $K=Z_{2} \times Z_{2}$. Suppose that $\operatorname{order}(z)=2$. Let $v$ be any other involution in $Z(G)$, and let $J=L\langle v\rangle$. By hypothesis, there is a conjugacy class $C$ of $G$ such that $C z J=C J$. Hence, $C z y=C=C(z y)^{3}$ for some $y \in J$. If $y \in L$, then $C=C(z y)^{3}=C z^{3}=C z$, as desired. If $y \in L v$, then $C=C(z y)^{3}=C z v$. It is assumed that there is an automorphism $\tau$ of $G$ with $(z v)^{\tau}=z$. Hence, $C^{\tau} z=C^{\tau}$ and the proof is finished.

Lemma 6. If $G$ is quasisimple, $G / Z(G) \cong \operatorname{PSU}\left(4,3^{2}\right)$ and $z \in Z(G)$ with $\operatorname{order}(z)=2,3$, or 4 , then $z$ fixes some conjugacy class of $G$.

Proof. Since $z$ is the image of an element of prime-power order in $Z(U)$, where $U$ is the universal covering group of $\operatorname{PSU}\left(4,3^{2}\right)$, and since $M\left(\operatorname{PSU}\left(4,3^{2}\right)\right) \cong Z_{4} \times Z_{3} \times Z_{3}$, it suffices to assume that $G=\mathrm{U}$. Let $Z(G)=L \times K, L \cong Z_{4}, K \cong Z_{3} \times Z_{3}$. From the description of the conjugacy classes of $G / K$ in the Atlas [CCNPW, p. 54], we see that if $z \in L$, then $z K$ fixes a conjugacy class of $G / K$. So $z$ fixes a conjugacy class of $G$, by Lemma 2.

There exists an automorphism $\tau$ of $G$ of order 4, which has two orbits of length 4 on $K-1$, and such that $\tau^{2}$ acts as inversion on $K$ [CCNPW, pp. 52,53]. So if $z \in K, z \neq 1$, and if $u=z^{\tau}$, then $K=\langle z\rangle \times\langle u\rangle$. Thus $K /\langle z\rangle, K /\langle u\rangle$ are groups denoted by $3_{i}, 3_{i}^{\prime}$ in the Atlas [CCNPW, p. 53], and $\left\{K /\langle z u\rangle, K /\left\langle z^{-1} u\right\rangle\right\}=\left\{3_{j}, 3_{j}^{\prime}\right\}$, where $\{i, j\}=\{1,2\}$. We may assume that $K /\langle z\rangle$ is $3_{1}$ or $3_{2}$.

Each $\chi \in \operatorname{Irr}(G / L)$ has kernel (the image of) exactly one of $G, K,\langle z\rangle,\langle u\rangle$, $\langle z u\rangle$, or $\left\langle z^{-1} u\right\rangle$, since $Z(G / \operatorname{ker} \chi)$ must be cyclic [I, (2.27)]. Exactly 20 characters $\chi \in \operatorname{Irr}(G / L)$ have kernel $G$ or $K$, and those $\chi$ with kernel $\varsubsetneqq K$ occur in complex conjugate pairs [CCNPW, p. 54].

Suppose that $K /\langle z\rangle=3_{1}$. Then 16 conjugate pairs of irreducible characters of $G / L$ have kernel $\langle z\rangle, 16$ pairs have kernel $\langle u\rangle, 13$ pairs have kernel $\langle z u\rangle$, and 13 have kernel $\left\langle z^{-1} u\right\rangle$ [CCNPW, pp. 54, 56, 58]. Let $\omega$ be a primitive complex cube root of 1 . Then

$$
\sum_{\chi \in \operatorname{Irr}(G / L)} \chi(z) / \chi(1)=20+32+16\left(\omega+\omega^{2}\right)+26\left(\omega+\omega^{2}\right)=10 .
$$

Suppose that $K /\langle z\rangle=3_{2}$. Then 13 conjugate pairs from $\operatorname{Irr}(G / L)$ have kernel $\langle z\rangle$ and another 13 pairs have kernel $\langle u\rangle$, while 16 pairs have kernel $\langle z u\rangle$, and 
another 16 have kernel $\left\langle z^{-1} u\right\rangle$. Hence,

$$
\sum_{\chi \in \operatorname{Irr}(G / L)} \chi(z) / \chi(1)=20+26+13\left(\omega+\omega^{2}\right)+32\left(\omega+\omega^{2}\right)=1 .
$$

Then $z$ fixes a conjugacy class of $G / L$, and hence of $G$, by Lemmas 1 and 2 . Proof of Theorem 1. Let $S=G / Z(G) ; S$ is nonabelian, of course, since $G$ is perfect. By the classification of the finite simple groups [G], and Lemmas 3 and 4 , we may assume that $S$ is one of $A_{6}, A_{7}$, a sporadic group, or a group of Lie type, defined in characteristic $p$, such that $p|| M(S) \mid$. (Note that $A_{5} \cong \operatorname{PSL}(2,5)$ is handled by Lemma 4.) When $M(S)$ is cyclic, all quasisimple $G$, where $G / Z(G)$ is in the finite set of these remaining groups $S$, are described in the Atlas. This includes $S=A_{6}, A_{7}$, and the sporadic groups. By examination of the lifting rows, we see that one of conclusions (i)-(iv) holds in each case.

So we may assume that $M(S)$ is noncyclic, and $S$ is one of $\operatorname{PSL}(3,4)$, $\mathrm{O}_{8}^{+}(2), \operatorname{PSU}\left(4,3^{2}\right), \operatorname{PSU}\left(6,2^{2}\right), \mathrm{Sz}(8)$, or ${ }^{2} E_{6}\left(2^{2}\right)$. If $\mathrm{U}$ is the universal cover of $\mathrm{Sz}(8)$ or of $\mathrm{O}_{8}^{+}(2)$, and $1 \neq z \in Z(\mathrm{U}) \cong Z_{2} \times Z_{2}$, then in any quasisimple homomorphic image of $U$ with cyclic center, the image of $z$ fixes a conjugacy class [CCNPW, pp. 28, 86]. Hence, the same is true for $z$, by Lemma 5. Thus, conclusion (iv) holds if $S \cong \mathrm{Sz}(8)$ or $\mathrm{O}_{8}^{+}(2)$.

If $S \cong \operatorname{PSU}\left(6,2^{2}\right)$ or ${ }^{2} E_{6}\left(2^{2}\right)$, then $M(S) \cong Z_{3} \times Z_{2} \times Z_{2}$. Suppose that $\mathrm{U}$ is the universal cover of $S, z \in Z(\mathrm{U})$ with $\operatorname{order}(z)=2$ or 3 , and $\overline{\mathrm{U}}$ is a homomorphic image of $U$ with $|Z(\bar{U})|=2$ or 3 . Then the Atlas [CCNPW, pp. $116,118,196]$ shows that $\bar{z}$ fixes a conjugacy class of $\bar{U}$, except that the case $S \cong{ }^{2} E_{6}\left(2^{2}\right)$ and $|Z(\overline{\mathrm{U}})|=3$ does not appear. But in this case, $\overline{\mathrm{U}}$ is the twisted Steinberg cover of $S$ (arising from a simply connected algebraic group). So the first part of the argument of Lemma 4 shows that here as well, $\bar{z}$ fixes a conjugacy class of $\overline{\mathrm{U}}$. Then Lemma 5 implies that conclusion (iv) holds for $z$ and $U$. So either (i) or (iv) is true when $S \cong \operatorname{PSU}\left(6,2^{2}\right)$ or ${ }^{2} E_{6}\left(2^{2}\right)$.

If $S \cong \mathrm{PSU}\left(4,3^{2}\right)$, then $M(S) \cong Z_{4} \times Z_{3} \times Z_{3}$. So either (ii) or (iv) holds, by Lemma 6 . If $S \cong \operatorname{PSL}(3,4)$, then $M(S) \cong Z_{3} \times Z_{4} \times Z_{4}$. So if $\operatorname{order}(z) \neq 3$ then (ii), (iii), or (iv) holds (again, the Atlas contains those cases where $Z(G)$ is cyclic). If $\operatorname{order}(z)=3$, then $Z(G) \cong\langle z\rangle \times K$, where $K$ is a 2-group. Lemma 2 implies that $z$ fixes a conjugacy class of $G$. The theorem is proved.

\section{ACKNOWLEDGMENT}

The author thanks Gary Seitz for his encouraging words.

ADDED IN PROOF

Richard Lyons has recently communicated to the author an outline of a proof that the 6-fold cover of ${ }^{2} E_{6}\left(2^{2}\right)$ indeed does not satisfy conclusion (iv) of Theorem 1.

\section{REFERENCES}

[ABEF] Z. Arad, H. I. Blau, J. Erez, and E. Fisman, Real table algebras and applications to finite groups of extended Camina-Frobenius type, J. Algebra (to appear).

[CCNPW] J. H. Conway, R. T. Curtis, S. P. Norton, R. A. Parker, and R. A. Wilson, Atlas of finite groups, Clarendon Press, Oxford, 1985. 
[FS] W. Feit and G. M. Seitz, On finite rational matrix groups and related topics, Illinois J. Math. 33 (1988), 103-131.

[I] I. M. Isaacs, Character theory of finite groups, Academic Press, New York, 1976.

[G] D. Gorenstein, Finite simple groups: An introduction to their classification, Plenum Press, New York, 1982.

[S1] I. Schur, Über die Darstellung der symmetrischen und der alternierenden Gruppe durch gebrochene lineare Substitutionen, J. Reine Angew. Math. 139 (1911), 155-250.

[S2] _ Gesammelte Abhandlungen, Springer-Verlag, Berlin and New York, 1973.

[SS] T. A. Springer and R. Steinberg, Conjugacy classes, Seminar on Algebraic Groups and Related Finite Groups, Lecture Notes in Math., vol. 131, Springer-Verlag, Berlin and New York, 1970.

[T] R. C. Thompson, Commutators in the special and general linear groups, Trans. Amer. Math. Soc. 101 (1961), 16-33.

Department of Mathematical Sciences, Northern Illinois University, DeKalb, IlliNOIS 60115

E-mail address: blau@math.niu.edu 